\title{
Antihyperglycemic, insulin-sensitivity and antihyperlipidemic potential of Ganoderma lucidum, a dietary mushroom, on alloxan- and glucocorticoid- induced diabetic Long-Evans rats
}

\author{
Md. Moklesur Rahman Sarker, ${ }^{1,2} *$ M.A.T.R. Zihad, ${ }^{1}$ Mohasiul Islam, ${ }^{1}$ Mazmun Nahar, ${ }^{1}$ \\ Md. Mahfuzul Islam, ${ }^{1}$ Hasan Imam, ${ }^{1}$ Ahandra Ghosh, ${ }^{1}$ Mohd Shahimi Mustapha, ${ }^{2}$ and \\ Nahlah Elkudssiah Ismail ${ }^{2}$
}

${ }^{1}$ Department of Pharmacy, School of Science, Primeasia University, HBR Tower, 9 Banani C/A, Dhaka-1213, Bangladesh; ${ }^{2}$ Faculty of Pharmacy, Lincoln University College, No. 2, Jalan Stadium, SS 7/15, Kelana Jaya, 47301 Petaling Jaya, Selangor, Malaysia

*Corresponding Author: Md. Moklesur Rahman Sarker, Ph.D., Associate Professor and Head, Department of Pharmacology, Faculty of Pharmacy, Lincoln University College, No. 2, Jalan Stadium, SS 7/15, Kelana Jaya, 47301 Petaling Jaya, Selangor, Malaysia

Running Title: Antihyperglycemic and antihyperlipidemic potential of Reishi mushroom

Submission Date: August 23, 2015, Acceptance date: December 29, 2015: Publication date: December 31, 2015

\begin{abstract}
Background: Reishi (Ganoderma lucidum) is a well-known and popular edible mushroom eaten all over the world. It has been used as an alternative medicine for many years in China, Korea, Japan, Malaysia, and in eastern Russia. This mushroom is reported to exhibit a number of medicinal properties including antitumor, antioxidant, immunomodulating, anti-inflammatory, hepatoprotective, and hypoglycemic activities, due to the presence of bioactive polysaccharide. Glucocorticoids, which are usually prescribed for the treatment of arthritis to protect inflammation and reduce pain, can induce hyperglycemia or aggravate the hyperglycemic condition. For example, causing very high glucose levels in diabetic patients. However, no report has been published for its effect on glucocorticoid-induced diabetes.
\end{abstract}

Objective: To investigate the effect of Ganoderma lucidum on alloxan- and glucocorticoidinduced diabetes in Long-Evans rats.

Methods: Alloxan monohydrate $(150 \mathrm{mg} / \mathrm{kg})$ was intraperitoneally administered to Long-Evans rats as a single dose. The same volume of normal saline was injected to control rats. Three days after alloxan injection, rats with plasma glucose levels higher than $12 \mathrm{mmoL} / \mathrm{L}$ were identified as diabetic and included in the study. Reishi mushroom was collected from the Mushroom Development Institute, Ministry of Agriculture, Savar, Dhaka, Bangladesh, where it was 
identified by a Taxonomist. Petroleum ether extract (PEE), known as petroleum spirit in the USA, and Methanol extract (ME) were prepared by maceration and distillation techniques. The extracts were orally administered once a day at doses of 200, 400, 600 and $800 \mathrm{mg} / \mathrm{kg}$, respectively for 7 days. Metformin $(150 \mathrm{mg} / \mathrm{kg}$ ) was orally administered as a standard antidiabetic drug. Glucose levels were measured at 0 and 7 th days of treatment. The rats were allowed to rest for 1 week without treatment. The animals were again injected with dexamethasone $(2 \mathrm{mg} / \mathrm{kg}$ ) through intra-muscular route for 3 days and glucose levels were monitored regularly. Rats were then further treated with PEE and ME and metformin for another 7 days and glucose levels were determined at 0 and 7 th days of treatment.

Results: The PEE and ME of Reishi mushroom dose-dependently reduced the plasma glucose levels in alloxan-and steroid-induced fasting diabetic rats. The maximum reduction of fasting plasma glucose levels observed by PEE $(800 \mathrm{mg} / \mathrm{kg})$ and $\mathrm{ME}(800 \mathrm{mg} / \mathrm{kg})$ were $55.57 \%$ and $36.01 \%$ in alloxan-induced and $51.41 \%$ and $32.02 \%$ in steroid-induced diabetic rats, respectively. On the other hand, metformin $(150 \mathrm{mg} / \mathrm{kg})$ resulted in the diminution of fasting blood glucose levels by 60.02 and $51.12 \%$ in the alloxan- induced and steroid-induced diabetic rats respectively. Both the PEE $(800 \mathrm{mg} / \mathrm{kg})$ and $\mathrm{ME}(800 \mathrm{mg} / \mathrm{kg})$ significantly augmented plasma insulin levels $(* * * \mathrm{P}<0.001$ and $* * \mathrm{P}<0.01)$ and reduced HbA1c $(* * \mathrm{P}<0.01$ and $* \mathrm{P}<$ $0.05)$ in alloxan-and steroid-induced diabetic rats. Additionally, treatment of diabetic rats with PEE $(800 \mathrm{mg} / \mathrm{kg})$ and ME $(800 \mathrm{mg} / \mathrm{kg})$ controlled the 2 -h post prandial elevated glucose levels in blood plasma. The same dose of the extracts also significantly reduced the levels of total cholesterol (TC) $(* * * \mathrm{P}<0.001$ and $* * * \mathrm{P}<0.01)$, triglyceride $(\mathrm{TG})(* * * \mathrm{P}<0.001$ and $* * \mathrm{P}<$ $0.01)$ and low-density lipoprotein-cholesterol (LDL-c) $(* * * \mathrm{P}<0.001$ and $* * * \mathrm{P}<0.001)$, as well as increased the level of high density lipoprotein cholesterol (HDL-c) $(* * \mathrm{P}<0.01$ and $* * \mathrm{P}<$ 0.01 , respectively).

Conclusion: Our study demonstrated that edible mushrooms-Reishi has antihyperglycemic, insulin-sensitivity, and hyperlipidemic effects against both alloxan and corticosteroid-induced diabetes rats. The bioactive chemicals responsible for those activities are most probably the polysaccharides available in the mushroom. Therefore, usage of Reishi mushrooms as vegetables or as extract will be beneficial for the management of diabetes.

Keywords: Antihyperglycemic, antidiabetic, insulin-sensitizer, anti-hyperlipidemic, Gano mushroom, Reishi mushroom, Ganoderma lucidum, Lingzhi, alloxan-induced diabetes, Steroid induced diabetes, Glycated haemolgobin (HbA1c), Oral glucose tolerance test (OGTT), Hyperlipidemia

\section{INTRODUCTION}

Diabetes mellitus is a metabolic disorder characterized by hyperglycemia and alterations in carbohydrate, protein and fat metabolism, which occur due to inadequate levels of or absence of insulin production by the beta-cells of the pancreas or/and resistance of the secreted insulin [1, 2]. Diabetes mellitus is associated with the risks of cardiovascular diseases, nephropathy, 
neuropathy, vision problem, weight gain, cancers and ultimate death of the patients [3-5]. The prevalence and incidence of diabetes is increasing all over the world [6]. According to the World Health Organization (WHO) report, 347 million people have diabetes worldwide and diabetic death will be projected to rise more than $50 \%$ in the next 10 years [7]. Although many synthetic oral anti-diabetic drugs are available, these drugs are also associated with the increased risk of cardiovascular diseases [8], renal impairments [9] and several types of cancers [10] as well. The limitations of modern synthetic antidiabetic drugs necessitate the investigation and use of alternative source of antidiabetic drugs that may be effective for glycemic control, without or with minimum adverse events. Functional foods, especially edible medicinal mushrooms, recently have attracted the attention of the medical and biological researchers, due to the fact that these agents are the most convenient means for the effective prevention and treatment of diseases without negligible or adverse effects.

Ganoderma lucidum (GL), known as Reishi mushroom or Lingzhi mushroom, called the bitter mushroom because of its bitter taste, is a fungus which has been used for thousands of years as health tonic to increase longevity [2,11, 12]. Traditionally, it has long been used to prevent and treat various diseases including cancer, diabetes, hypertension, chronic bronchitis, hepatitis and infections in countries like China, Japan, Russia and Korea [13]. In the meantime, a number of bioactive ingredients, such as, ganoderic acid (triterpenoids), polysaccharides, nucleosides, adenosine, sterols, and alkaloids have been isolated from this mushroom, while polysaccharides are the main therapeutic chemicals [11,13]. Lansotane triterpenes isolated from Ganoderma lucidum were reported to possess anti-inflammatory and heme oxygenase-1 activities in RAW 264.7 cells [14].

Crude aqueous extract and proteoglycan from Ganoderma lucidum fruiting bodies showed anti-diabetic, antihyperlipidemic and antioxidant activities on mice $[12,15,16]$. The aqueous extract of this mushroom exhibited anticonvulsant, antidepressant and anxiolytic activities in experimental mice and rats [17, 18], whereas ethanol extract showed antimalarial and hepatoprotective effects in experimental mice [19]. It has been reported that the antimicrobial activity of GL is caused by the presence of triterpenoid and steroid compounds [20]. GL and its polysaccharides were reported to possess immunomodulatory [21-23], effect anti-angiogenic, and chemo-preventive or anticancer activities against a number of cancers [13, 24-26]. Some researchers reported the antidiabetic activities of GL and its isolated biochemicals, such as polysaccharides, proteoglycan [27-29], still extensive studies for the anti-hyperglycemic activities of GL and its different biochemicals are needed.

The dose of Ganoderma lucidum depends on its content of polysaccharide peptides and triterpenes. The Ganopoly (a Ganoderma lucidum polysaccharide extract) was used in some clinical trials, with dosages up to $5.4 \mathrm{~g} /$ day [30]. The State Pharmacopoeia Commission of the People's Republic of China recommends 6-12 $\mathrm{g}$ of this mushroom extract daily [31]. In an acute toxicological study with rats, Ganoderma lucidum extract/polysaccharide oral doses of up to 5 $\mathrm{g} / \mathrm{kg}$ body weight did not cause behavioral changes and no death was observed [2]. A study also reported that oral consumption of $1.44 \mathrm{~g}$ of Ganoderma extract for 28 days did not exhibit any toxicological signs in liver, renal, heart and blood parameters [32]. 
The objective of the present study was to evaluate the antihyperglycemic and insulin sensitivity potential of petroleum ether extract (PEE) and methanol extract (ME) of GL on alloxan- as well as corticosteroid-induced diabetic Long-Evans rats.

\section{MATERIALS AND METHODS}

Collection of mushroom: Fresh Reishi mushroom (Ganoderma lucidum) was collected from the Mushroom Development Institute, Ministry of Agriculture, Sobhanbag Savar Dhaka, Bangladesh, where it was identified by a Taxonomist and a voucher specimen was retained for further reference.

Preparation of extract: The mushrooms were cut into small pieces and shed dried with a good air circulating system for 2 weeks. The dried mushrooms were grinded to coarse powder with a mechanical grinder (multi-mill) and screened through mesh to remove large particles. The extracts were prepared by using maceration technique [33]. Twenty gram of mushroom powder was soaked in $200 \mathrm{~mL}$ of $99.8 \%$ methanol (chemical ID: 106009 ; boiling point range: $64-65^{\circ} \mathrm{C}$; Merck, Germany) and $99.7 \%$ of petroleum ether (chemical ID: 101769; boiling point range: 40$80^{\circ} \mathrm{C}$; Merck, Germany) separately at room temperature for 2 days with occasional shaking. The extracted solution was then filtered with Whatman No. 1 and stored at refrigerator. The extraction process was repeated 5 times with the incorporation of the same solvent until the extract depicts no faint discoloration and the filtrates were accumulated in a beaker [33]. PEE and ME of GL were then concentrated for complete removal of extra solvents under reduced pressure by using a rotary evaporator at $40^{\circ} \mathrm{C}$ [34]. The yields of PEE and ME were 17.05 (3.41 g) and $12.35 \%(2.47 \mathrm{~g})$ respectively. The extracts were stored at $4{ }^{\circ} \mathrm{C}$ in a refrigerator until use.

Experimental animals: Long-Evans male rats (4 weeks age, weight $83.5 \mathrm{~g}$ ) were purchased from the Animal Resource Branch of International Centre for Diarrheal Disease Research in Bangladesh (ICDDRB). The animals were kept and maintained under standard environmental conditions (temperature at $25 \pm 5^{\circ} \mathrm{C}$, relative humidity 40-60\%, 12 hour dark and light cycle), and fed ad libitum with normal animal pellet diet. The animals were allowed to acclimatize for 2 weeks before commencing the experiments. All the studies were conducted according to the guidelines of Animal Ethical Committee of Primeasia University, Dhaka, Bangladesh.

Induction of diabetes by Alloxan monohydrate: Diabetes was induced to Long-Evans rats by intraperitoneal administration of a single dose of Alloxan monohydrate $(150 \mathrm{mg} / \mathrm{kg})$ [35]. Blood glucose levels were monitored each day using a glucometer (Accu-Chek, Roche). Three days after alloxan injection, rats with plasma glucose levels higher than $12 \mathrm{mmoL} / \mathrm{L}$ were considered as diabetic rats and they were included in the study.

Induction of diabetes by Corticosteroid (Dexamethasone): At the end of first round treatment of alloxan-induced diabetic rats with GL extracts, the rats were allowed to rest for 1 week without treatment. Diabetes was again induced in the rats with intra-muscular injection of dexamethasone ( $3 \mathrm{mg} / \mathrm{kg} / \mathrm{day}$ ) [36] for 3 consecutive days. Glucose levels were measured and we 
discovered that the plasma glucose levels of all rats were potentially elevated. The glucose levels of all the rats were found to be higher than $12 \mathrm{mmoL} / \mathrm{L}$ and they were used for further treatment with the extracts.

Experimental Design: A total of 55 male Long-Evans rats were used for this study. The animals were randomly divided into the following groups:

Group I: Nondiabetic rats

Group II: Diabetic rats (control)

Group III: Diabetic rats treated with GL-ME $(200 \mathrm{mg} / \mathrm{kg})$

Group IV: Diabetic rats treated with GL-ME $(400 \mathrm{mg} / \mathrm{kg})$

Group V: Diabetic rats treated with GL-ME $(600 \mathrm{mg} / \mathrm{kg})$

Group VI: Diabetic rats treated with GL-ME $(800 \mathrm{mg} / \mathrm{kg})$

Group VII: Diabetic rats treated with GL-PEE $(200 \mathrm{mg} / \mathrm{kg})$

Group VIII: Diabetic rats treated with GL-PEE $(400 \mathrm{mg} / \mathrm{kg})$

Group IX: Diabetic rats treated with GL-PEE $(600 \mathrm{mg} / \mathrm{kg})$

Group X: Diabetic rats treated with GL-PEE $(800 \mathrm{mg} / \mathrm{kg})$

Group XI: Diabetic rats treated with Metformin $(150 \mathrm{mg} / \mathrm{kg})$

The extracts and metformin were administered to the respective groups by oral gavage using stainless steel feeding needle. Normal saline was used as a vehicle of extract for administration; control group was administered with the same volume of saline only. The animals were sacrificed at the end of the experiments and the collected serum was used for the determination of glucose, insulin, and lipid levels.

Oral Glucose Tolerance Test (OGTT): Oral glucose tolerance test was performed as described by Kumar et al [34]. Alloxan-induced diabetic rats were treated with ME and PEE regularly. At day 7, overnight fasted (14 hours) diabetic Long-Evans rats were treated with ME or PEE or metformin or with normal saline. Glucose $(2 \mathrm{~g} / \mathrm{kg})$ was fed 30 minutes after the administration of extracts or metformin doses. Blood was collected from the tail vein to measure the glucose levels at 0 and 2 hours of glucose administration. Similarly, OGTT was performed to dexamethasone induced diabetic rats at day 7 of treatment, using the method described above.

Determination of fasting glucose, insulin and HbA1c in blood serum: Alloxan-induced diabetic rats were administered with different doses of ME and PEE of GL or metformin daily for 7 days, similar to standards of other researchers [37,38]. The animals were kept at rest for 1 week. Dexamethasone ( $3 \mathrm{mg} / \mathrm{kg} / \mathrm{day}$ ) was injected for 3 consecutive days to induce diabetes or elevate the glucose levels. Dexamethasone-induced diabetic rats were again treated with GLextracts and metformin for another 7 days. Blood samples were collected from tail vein of overnight fasting rats on days 0 and 7 (alloxan-induced diabetic rats), and 18 and 25 (dexamethasone-induced diabetic rats). Serum glucose level was measured by Glucose oxidase method [39] and serum insulin level was assayed by using an Enzyme Linked Immunosorbant 
Assay (ELISA) kit [35]. Glycated haemoglobin (HbA1c) was estimated by a colorimetric method with some modification [35].

Measurement of serum lipid levels: On day 25, overnight fasted rats were anesthetized by ether and blood samples were collected by cardiac puncture. The blood was then centrifuged at $1500 \times$ $\mathrm{g}$ for $15 \mathrm{~min}$ at $4{ }^{\circ} \mathrm{C}$ to collect the supernatants for the determination of lipid levels. Triglycerides (TG) [6], total cholesterol (TC), low density lipoprotein cholesterol (LDL-c), and high density lipoprotein cholesterol (HDL-c) were determined by using a bioanalyzer with standard kits [39].

Statistical analysis: The data was analyzed by SPSS software package Version 22.0. The values were analyzed by one-way analysis of variance (ANOVA) followed by the Tukey's post hoc test. The data are means \pm standard error of mean $(\mathrm{SEM})$. $\mathrm{P}$ values less than $0.05(\mathrm{P}<0.05)$ were considered as significant.

\section{RESULTS}

Reduction of fasting plasma glucose by petroleum ether and methanol extracts of Ganoderma lucidum: The effects of oral administration of PEE and ME of GL on alloxan- and steroid-induced diabetic Long-Evans rats were presented in Fig. 1.

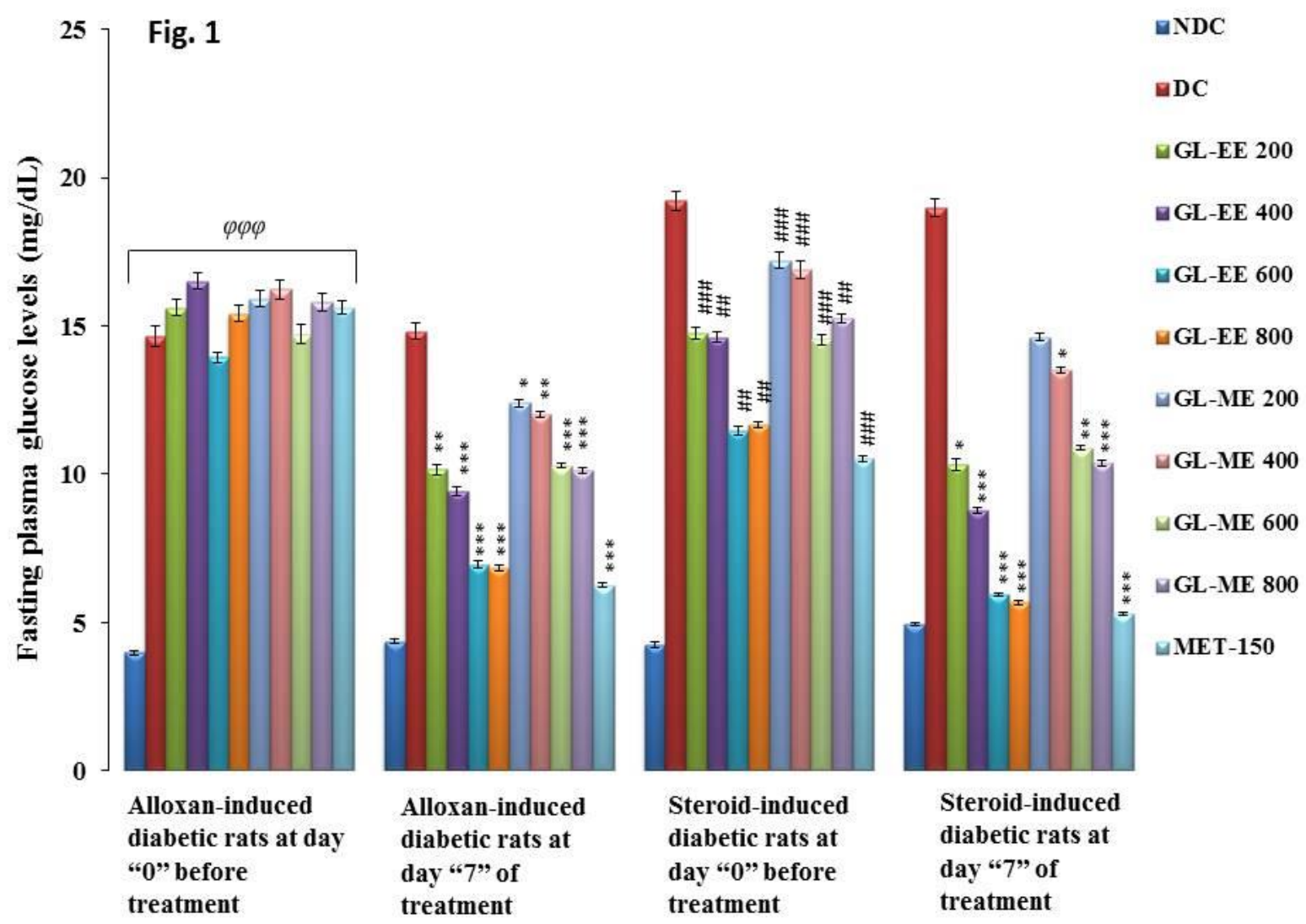

Fig 1: Effect of GL-PEE on fasting plasma glucose levels in alloxan- and steroid-induced 
diabetes Long-Evans rats. The rats were induced to diabetes with the i.p. injection of a single dose of alloxan $(150 \mathrm{mg} / \mathrm{kg})$. The animals were then treated with GL-PEE and GL-ME for 7 days and plasma glucose levels were measured at day 0 and 7 of treatment. Following 1 week resting time, rats were injected with dexamethasone for 3 consecutive days and again treated with extracts for 7 days to measure plasma glucose levels at day 0 and 7 . The data are means \pm S.E.M. of 5 rats in a group, $* \mathrm{P}<0.05$; $* * \mathrm{P}<0.01$; $* * * \mathrm{P}<0.001$ (post- versus pre-treatment in alloxaninduced or steroid-induced diabetic rats); \#\# $\mathrm{P}<0.01$, \#\# $\mathrm{P}<0.001$ (glucose level change in post- versus pre-steroid-treatment); and ${ }^{\varphi \varphi} \mathrm{P}<0.001$ (alloxan-induced diabetic vs. non-diabetic rats group) (Tukey's HSD test). $[\mathrm{NDC}=$ Non-diabetic control, DC $=$ Diabetic control, GL-EE $200=$ Diabetic rats treated with ether extract of GL (200 mg/kg), GL-EE 400/600/800 = Diabetic rats treated with ether extract of GL 400/600/800 mg/kg, GL-ME $200=$ Diabetic rats treated with methanol extract of GL (200 mg/kg), GL-ME 400/600/800 = Diabetic rats treated with methanol extract of GL 400/600/800 mg/kg, and MET-150 = Diabetic rats treated with metformin (150 $\mathrm{mg} / \mathrm{kg})]$

Injection of a single dose of Alloxan (150 mg/kg, i.p.) induced diabetes in Long-Evans rats with significant changes in fasting blood glucose levels compared to non-injected rats $(\varphi \varphi \varphi \mathrm{P}<0.001)$. The treatment of alloxan-induced diabetic rats with PEE and ME for 7 days dose-dependently reduced fasting plasma glucose levels comparing post- versus pre-treatment diabetic groups (*P $<0.05, * * \mathrm{P}<0.01$, and $* * * \mathrm{P}<0.001)$. The maximum reduction of fasting plasma glucose levels observed on day 7 were $55.57 \%$ and $36.01 \%$ by PEE $(800 \mathrm{mg} / \mathrm{kg})$ and $\mathrm{ME}(800 \mathrm{mg} / \mathrm{kg})$ respectively. Following 1 week of resting time after extract treatment, the rats were injected with dexamethasone for 3 consecutive days, which resulted in significant elevation of glucose levels in diabetic rats $(\# \# \mathrm{P}<0.01$, \#\# $\mathrm{P}<0.001)$. Further treatment of steroid-induced diabetic rats with extracts for further 7 days resulted in the dose-dependent significant reduction of glucose levels by both the PEE and ME of GL ( $* \mathrm{P}<0.05$; **P $<0.01$; ***P $<0.001$; compared to postversus pre-treatment groups). The highest glucose lowering effects in steroid-induced diabetic rats were $51.41 \%$ and $32.02 \%$ by $\mathrm{PEE}(800 \mathrm{mg} / \mathrm{kg})$ and $\mathrm{ME}(800 \mathrm{mg} / \mathrm{kg})$, respectively, comparing post- versus pre-treatment of the same rat groups. The glucose reducing ability of extracts were compared with a standard antidiabetic drug, metformin $150 \mathrm{mg} / \mathrm{kg}$, which resulted in the diminution of fasting blood glucose levels by 60.02 and $51.12 \%$ in the alloxan-induced and steroid-induced diabetic rats, respectively.

Petroleum ether and methanol extracts of Ganoderma lucidum increased blood insulin levels: The effects of oral administration of PEE and ME of GL is presented in Fig 2. There was a significant decline in plasma insulin levels in alloxan-induced diabetic rats as compared to noninduced control rat group ( ${ }^{\varphi \varphi \varphi} \mathrm{P}<0.001$ ). Treatment of alloxan-induced diabetic rats significantly elevated the plasma glucose levels when compared post-versus pre-treatment rats of the same group ( $* * \mathrm{P}<0.01, * * * \mathrm{P}<0.001$ ). PEE and ME at a dose of $800 \mathrm{mg} / \mathrm{kg}$ increased plasma insulin levels in diabetic rats by $78.34 \%$ and $52.08 \%$, respectively. Metformin, on the contrary, at a dose of $150 \mathrm{mg} / \mathrm{kg}$ could not bring out significant changes in plasma insulin level in diabetic rats (Fig. 2). 
Fig. 2

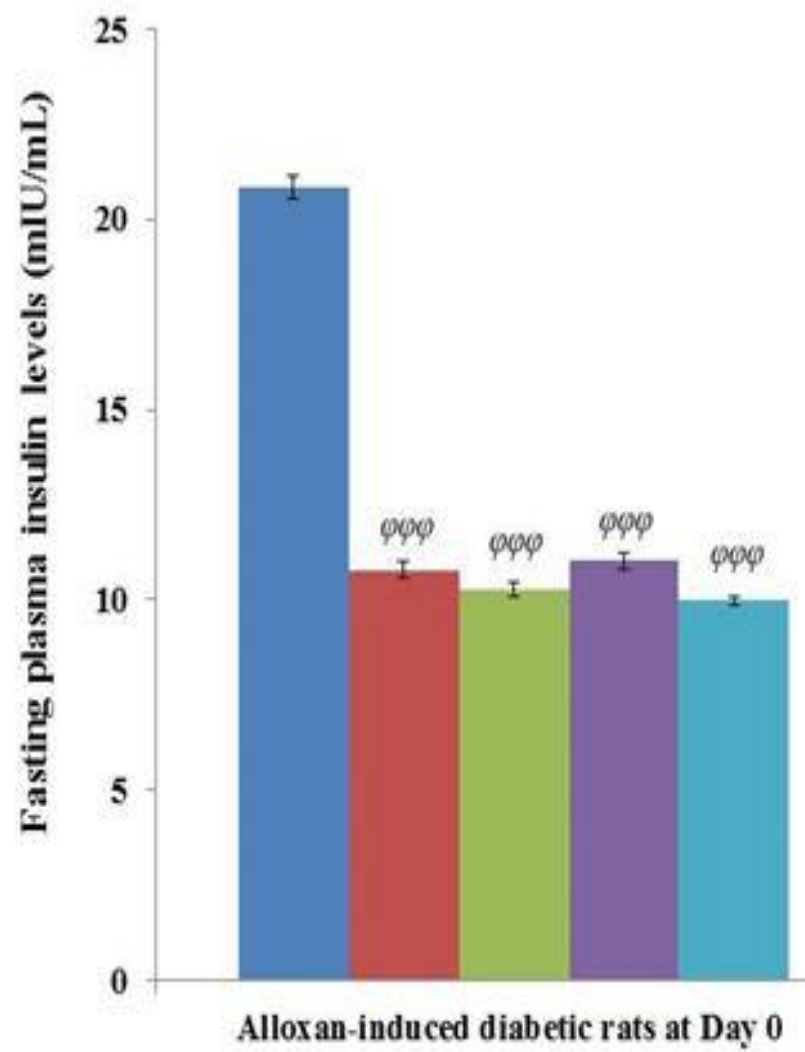

Alloxan-induced diabetic rats at Day 0
= Nondiabetic control

- Diabetic control

= Pet-Ether Extract $800 \mathrm{mg}$ treated diabetic rats Meth anol extract $800 \mathrm{mg}$ treated diabetic rats

$=$ Metformin $150 \mathrm{mg}$ treated diabetic rats

Fig 2: Effect of GL extracts on fasting plasma insulin levels in alloxan- and steroid-induced diabetes Long-Evans rats. Rats were induced to diabetes with the i.p. injection of a single dose of alloxan $(150 \mathrm{mg} / \mathrm{kg})$. The animals were treated with GL-PEE and GL-ME for 7 days and plasma insulin levels were measured at day 0 and 7 of treatment. The data are means \pm S.E.M. of 5 rats in a group, $* * \mathrm{P}<0.01$ and $* * * \mathrm{P}<0.001$ (post- vs. pre-treatment groups); and ${ }^{\varphi \varphi \varphi} \mathrm{P}<0.001$ (alloxan-induced diabetic vs. non-diabetic rats group) (Tukey's HSD test).

\section{Reduction of glycated hemoglobin (HbA1c) by GL-PEE and GL-ME in diabetic rats:}

Administration of a single dose of alloxan $(150 \mathrm{mg} / \mathrm{kg}$, i.p.) induced diabetes and significantly elevated HbA1c (\%) 3 days after the challenged compared to non-induced rats (\#\#\# $\mathrm{P}<0.001$ ). Treatment of alloxan-induced (7 days) and steroid-induced diabetic rats (7 days) with GL-PEE $(800 \mathrm{mg} / \mathrm{kg})$, GL-ME $(800 \mathrm{mg} / \mathrm{kg})$, and metformin $(150 \mathrm{mg} / \mathrm{kg})$ significantly reduced HbA1c levels $(\%)$ compared to post- versus pre-treatment status of the same rat groups $(* \mathrm{P}<0.05$, **P $<0.01)$ 
Fig. 3

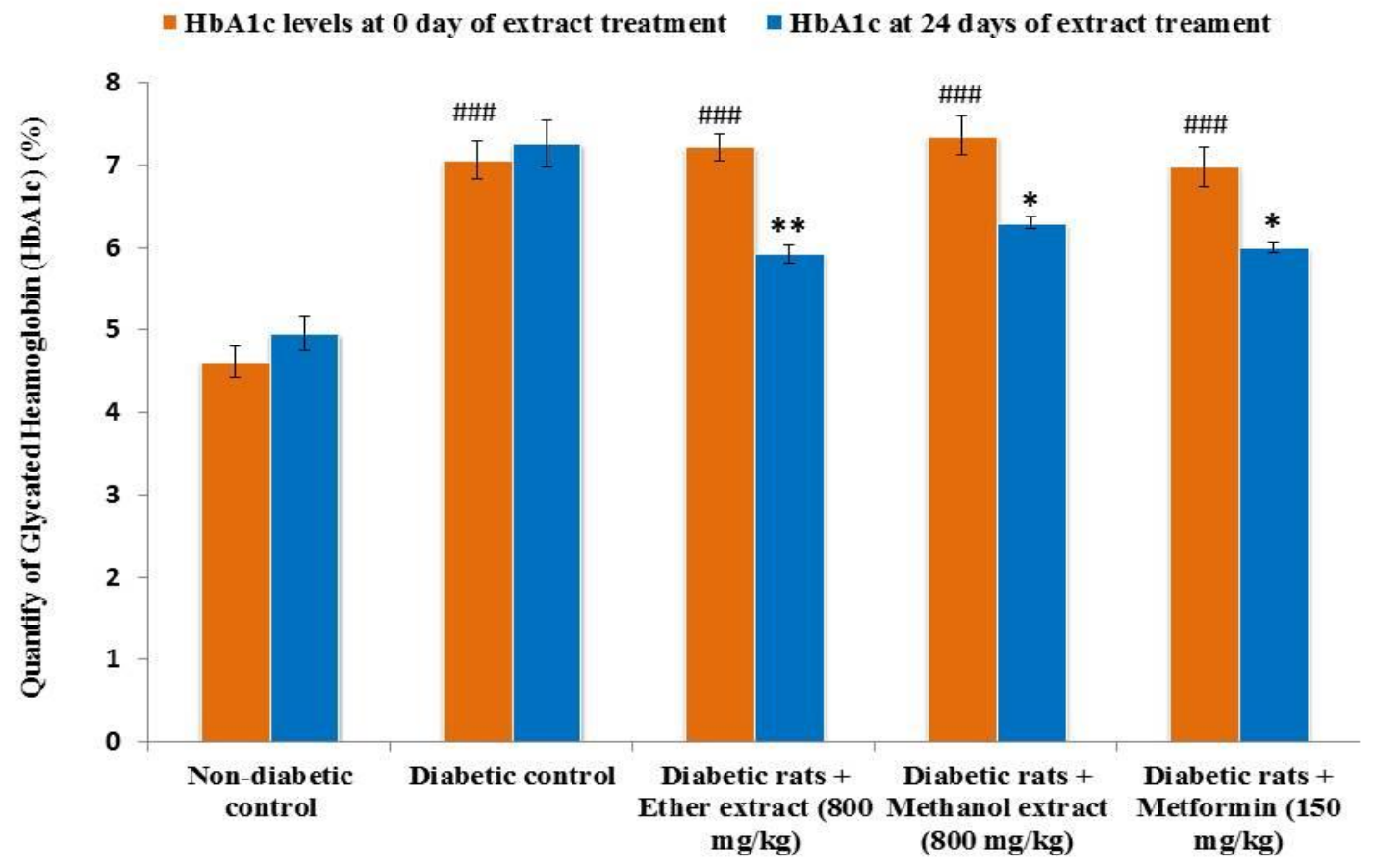

Fig 3: Effect of GL extracts on HbA1c levels in alloxan- and steroid-induced diabetes LongEvans rats. The rats were induced to diabetes with the i.p. injection of a single dose of alloxan $(150 \mathrm{mg} / \mathrm{kg})$ and were treated with GL-PEE and GL-ME for 7 days. Following 1 week resting time, animals were injected with dexamethasone for 3 consecutive days and again treated with extracts for 7 days. On day 25, the rats were sacrificed. Plasma HbA1c levels were determined in fasting rats at day 0 and 25 days of study. The data are means \pm S.E.M. of 5 rats in a group; \#\#\# $\mathrm{P}<0.001$ (HbA1c change in alloxan-induced vs. non-induced rats groups at day 0 of treatment); $* \mathrm{P}<0.05$; ** $\mathrm{P}<0.01$ (Change in HbA1c levels in diabetic rats on day 25 vs. day 0 of extract treatment) (Tukey's HSD test).

Improvement of post-prandial glucose homeostasis by GL extracts: The effects of PEE and ME $(800 \mathrm{mg} / \mathrm{kg}$ ) were evaluated for oral glucose tolerability test (OGTT) on non-diabetic and alloxan-induced diabetic rats. The extracts were orally administered to the rats 30 minutes before glucose loading and the post-prandial glucose levels were measured 2 hours after glucose administration. Both the PEE and ME promoted glucose uptake and maintained the glucose homeostasis by preventing the significant raising of plasma glucose levels compared to postversus pre-prandial plasma glucose levels. The 2-h post-prandial plasma glucose level of untreated diabetic rats significantly increased compared to pre-prandial glucose levels (***P < 0.001). When compared with the diabetic controlled rats, the GL-PEE ( $\left.\varphi^{\varphi} \varphi \mathrm{P}<0.001\right)$, and GL$\operatorname{ME}(\varphi \varphi \varphi \quad \mathrm{P}<0.001)$, and metformin $(\varphi \varphi \varphi \mathrm{P}<0.001)$ treatment significantly reduced 2-h postprandial plasma glucose levels in diabetic rats (Fig. 4). 
Fig. 4

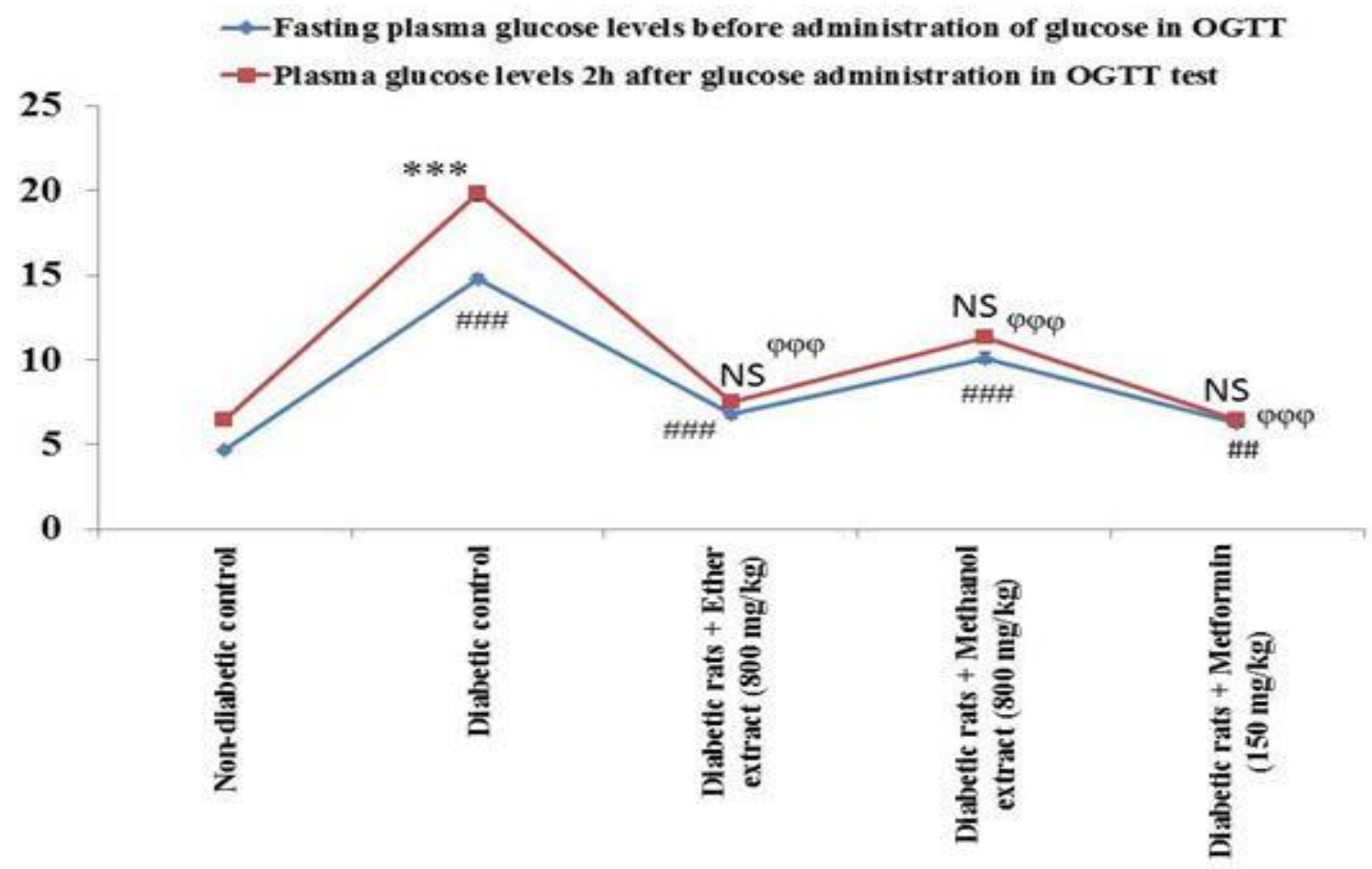

Fig 4: Effect of GL extracts on OGTT in diabetes Long-Evans rats. The rats were induced to diabetes with the i.p. injection of a single dose of alloxan $(150 \mathrm{mg} / \mathrm{kg})$. The animals were then treated with methanol and petroleum ether extracts of GL for 7 days. At day 7, overnight fasted (14 hours) diabetic rats were treated with GL-PEE or GL-ME or metformin or with normal saline. Glucose (2g/kg) was fed 30 minutes after treatment. Plasma glucose levels were measured in blood from tail vein at 0 and 2 hours of glucose administration. The data are means \pm S.E.M. of 5 rats in a group. \#\# $\mathrm{P}<0.01$, \#\#\# $\mathrm{P}<$ 0.001 (Plasma glucose levels in diabetic vs. non-diabetic rats before oral administration of glucose); ***P $<0.001$ (Plasma glucose levels at 2 hours post- versus pre-administration of oral glucose in diabetic untreated rats group); ${ }^{\varphi \varphi \varphi} \mathrm{P}<0.001$ (Change in plasma glucose levels in treated vs. untreated diabetic rats at 2 hours after the administration of oral glucose) (Tukey's HSD test). NS means non-significant changes in plasma glucose level in diabetic treated rats at 2 hours after the administration of oral glucose compared to the glucose levels measured before the administration of oral glucose.

Effect of Ganoderma lucidum on lipid profile in diabetic rats: The effect of GL-PEE and GLME on lipid profile of diabetic rats is presented in Table 1. After 24 days study period the overnight fasting $(14 \mathrm{~h}$ ) rats were sacrificed on day 25 and collected blood was analyzed for lipid profile. As shown in the Table 1, the levels of TC, TG and LDL-c were significantly higher (\#\#\# $\mathrm{P}<0.001$, \#\# $\mathrm{P}<0.001$ and \#\# $\mathrm{P}<0.01$, respectively) and HDL-c level was significantly lower (\#\#\# $\mathrm{P}<0.001$ ) in diabetic rats compared to nondiabetic rats. Treatment of diabetic rats with GLPEE $(800 \mathrm{mg} / \mathrm{kg})$ significantly reduced TC $(* * * \mathrm{P}<0.001)$, TG $(* * * \mathrm{P}<0.001)$ and LDL-c $(* * * \mathrm{P}<0.001)$ levels, and HDL-c level was significantly increased $(* * \mathrm{P}<0.01)$ compared to diabetic control rats. Similarly, GL-ME significantly reduced TC, TG and LDL-c and increased HDL-c levels compared to untreated diabetic rats. Overall, the effect of GL-PEE was comparatively better than that of GL-ME. The effects of the extracts were comparable with the effect of metformin for controlling lipid profile. 
Table 1. Effect of GL-PEE and GL-ME on the serum lipid profile on alloxan- and steroidinduced diabetic rats.

\begin{tabular}{|c|c|c|c|c|}
\hline Treatment group & TC (mg/dL) & TG (mg/dL) & $\begin{array}{c}\text { LDL-c } \\
(\mathrm{mg} / \mathrm{dL})\end{array}$ & $\begin{array}{c}\text { HDL-c } \\
(\mathrm{mg} / \mathrm{dL})\end{array}$ \\
\hline Non-diabetic control & $102 \pm 2.156$ & $86 \pm 1.864$ & $43 \pm 1.869$ & $57 \pm 0.967$ \\
\hline Diabetic control & $198 \pm 2.563^{\# \# \#}$ & $170 \pm 2.652$ & $\underset{\# \#}{156 \pm 2.954}$ & $\underset{\text { \#\#\# }}{28 \pm 0.568}$ \\
\hline Diabetic rats + Ether extract $(800 \mathrm{mg} / \mathrm{kg})$ & $140 \pm 2.205^{* * *}$ & $124 \pm 2.325^{* * *}$ & $104 \underset{* * *}{ \pm 2.778}$ & $40 \pm \underset{* *}{0.744}$ \\
\hline $\begin{array}{l}\text { Diabetic rats }+ \text { Methanol extract }(800 \\
\mathrm{mg} / \mathrm{kg})\end{array}$ & $153 \pm 2.591^{* *}$ & $132 \pm 2.591^{* *}$ & $120 \pm 2.684$ & $37 \pm \underset{* *}{0.372}$ \\
\hline Diabetic rats + Metformin $(150 \mathrm{mg} / \mathrm{kg})$ & $132 \pm 2.887^{* * *}$ & $115 \pm 2.438^{* * *}$ & $75 \pm 1.948^{* * *}$ & $42 \pm \underset{* * *}{0.442}$ \\
\hline
\end{tabular}

Values are expressed as means \pm S.E.M., $\mathrm{n}=5$. \#\# $\mathrm{P}<0.01$, \#\#\# $\mathrm{P}<0.001$ comparison made between diabetic control and non-diabetic control groups; ${ }^{*} \mathrm{P}<0.01,{ }^{* * *} \mathrm{P}<0.001$ comparisons made between extract or metformin treated group and diabetic control groups.

\section{DISCUSSION}

The present study exertion was designed to evaluate the antihyperglycemic activity, insulin sensitivity, and lipid lowering effects petroleum ether and methanol extracts of Gano mushroom (Ganoderma lucidum) on alloxan-and steroid-induced diabetic Long-Evans rats.

Alloxan is a widely used chemical for the induction of diabetes in animal model [35]. Alloxan is a toxic glucose analogue which predominantly accumulate in beta cells of the pancreas via glucose transporter GLUT2 [40]. Alloxan produces reactive oxygen species (superoxide radicals, hydrogen peroxide, hydroxyl free radicals) which cause the death of betacells. Besides, alloxan has the ability to inhibit the beta-cell glucose-sensor glucokinase. Alloxan therefore selectively inhibits glucose-induced insulin secretion [40]. Consequently, alloxan results drastic reduction of insulin release from beta-cells which results hyperglycemia. In our study, diabetes was induced by the administration (i.p.) of a single dose a single dose (150 $\mathrm{mg} / \mathrm{kg}$ ) of alloxan to Long evans rats and hyperglycemia (plasma glucose level $\geq 12 \mathrm{mmoL} / \mathrm{dL}$ ) was confirmed on $3^{\text {rd }}$ day after alloxan administration.

Glucocorticoids, commonly used for the treatment of inflammation and arthritis [41], causes insulin-resistance, hyperglycemia, and hyperlipidemia [42]. Glucocorticoids increase adipocity and lipolysis which results high level of free fatty acids in the circulation. The free fatty acids cause insulin resistance. Glucocorticoids induce proteolysis and increased release of amino acids, which cause insulin resistance. The binding of insulin to its receptor (IR) causes increased kinase activity and tyrosine phosphorylation of several downstream signaling molecules including IRS1. Glucocorticoids decrease transcription of IRS-1 and increase the transcription of two proteins (tyrosine phosphatase type 1B and p38MAPK) that counter insulin action. Therefore, Glucocorticoid decreases insulin signaling and contributes to insulin resistance [42]. Insulinresponse to liver decrease/inhibit glycogenesis and gluconeogenesis, insulin thus decreases glucose output from liver. Glucocorticoids decrease the sensitivity of liver to insulin, which 
promote hepatic glucose output. These drugs inhibit glucose uptake by muscle and fat and inhibit $60 \%$ of insulin sensitivity [41]. Corticosteroids are prescribed for the treatment of arthritis. In case of elder patients, steroids can easily induce diabetes. Moreover, steroids may aggravate the diabetic conditions in patients with diabetes with the increase in hyperglycemia. In this study, a significant elevation of plasma glucose level was observed with the administration (i.m.) of dexamethasone (3 $\mathrm{mg} / \mathrm{kg} / \mathrm{day}$ ) on 3 consecutive days to Long evans rats. Treatment with Ganoderma lucidum petroleum ether extracts $(200-800 \mathrm{mg} / \mathrm{kg}$ ) and methanol extracts (400-800 $\mathrm{mg} / \mathrm{kg}$ ) significantly reduced steroid-induced hyperglycemia in diabetic rats (Fig. 1).

Fasting plasma glucose (FPG) is considered to be the main diagnostic test for the diabetes mellitus [43]. The high level of fasting plasma glucose may be result from the impaired sensitization of secreted insulin or the insufficient production of insulin for the sufficient uptake of glucose to the cells. The fasting plasma glucose (FPG) levels were highly increased in both the alloxan and steroid (dexamethasone) induced rats to make them diabetic compared to noninduced animal group (Fig. 1). Treatment of alloxan-induced and steroid-induced diabetic rats with both the PE and ME (200-800 mg/kg) extracts of Ganoderma lucidum potentially reduced FPG levels in diabetic rats. The possible mechanism behind the improvement of fasting plasma glucose levels by extract may be due to the stimulating effect of the extracts to the beta-cells of the pancreas for the enhanced secretion of insulin or protecting the beta-cells from the possible further degradation by alloxan toxicity or it plays a role for the repairmen of alloxan-impaired beta-cells health for sufficient secretion of insulin. In addition, the extracts may stimulate the sensitivity of secreted insulin which converts the inactive form of insulin to active for the enhanced uptake of glucose. Thus, the extracts contribute for the reduction of fasting hyperglycemia.

As shown in the Fig. 2, the levels of plasma insulin in alloxan- induced diabetes rats demonstrated remarkable decrease compared to non-diabetic rats. Treatment of diabetic rats with Ganoderma lucidum PE (800 mg/kg) and ME (800 mg/kg) remarkably improved plasma insulin levels. The possible mechanism may be the result of the beta-cells' health protection ability of the extracts by preventing glucotoxicity to the beta cells and repairing the impaired condition of beta-cells' health due to alloxan-treatment and providing a shield to the beta-cells for further deterioration. The biochemical of the extracts thus improves the protection and activity of betacells for continuous production of insulin.

Glycated hemoglobin (HbA1c) is a diagnostic marker which is found to be increased in diabetes over a long time. In chronic diabetes, the excess glucose in the blood reacts with haemoglobin to form glycosylated haemoglobin or simply called glycated haemoglobin, and the rate of $\mathrm{HbA} 1 \mathrm{c}$ formation is proportional to the concentrations of free blood glucose [35]. We observed that the level of HbA1c was significantly increased (\#\#\# $\mathrm{P}<0.001$ ) in alloxan- and steroid-induced diabetic rats compared to non-diabetic rats (Fig. 3). Treatment of diabetic rats with Ganoderma lucidum PE (800 mg/kg), ME (800 mg/kg), and metformin (150 mg/kg) significantly reduced the HbA1c levels $(* * \mathrm{P}<0.001$, $* * \mathrm{P}<0.01$ and $* * \mathrm{P}<0.01$, respectively). The effects of Ganoderma- PE and ME extracts $(800 \mathrm{mg} / \mathrm{kg}$, each) to reduce HbA1c was comparable to that of metformin (Fig. 3). The possible mechanism of action for the reduction of HbA1c level 
by extracts is that with the reduction of plasma glucose levels in the blood, mushroom extracts reduced plasma $\mathrm{HbA1c}$ levels.

Oral glucose tolerance test is used to evaluate the body's ability to use glucose. After oral glucose administration, the glucose uptake by the cells mainly depends on the secretion and sensitivity of insulin from the pancreatic beta cells. In our experiments, elevated glucose levels were observed in alloxan induced diabetic rats compared to non-diabetic rats (Fig. 4). On the contrary, the level of plasma insulin was decreased in diabetic rats. Oral administration of different doses of Ganoderma lucidum petroleum ether $(200,400,600$, and $800 \mathrm{mg} / \mathrm{kg}$ ) and methanol extracts $(400,600$, and $800 \mathrm{mg} / \mathrm{kg})$ potentially reduced plasma glucose levels with the improvement of plasma insulin levels in the alloxan-induced diabetic rats. Our result suggests that rats treated with Gano muashroom extracts have better glucose utilization capacity, which may be due to increased insulin emission from $\beta$-cells and improved glucose transport and consumption. Many plant extracts or derived chemicals have been reported to exhibit antihyperglycemic activity through the stimulation of insulin secretion [33, 44].

Hyperlipidemia increases the risk of cardiovascular diseases, the leading cause of death all over the world [45]. Moreover, there is a strong and long-lasting relationship between high level of triglycerides and the development of cardiovascular diseases [46] including coronary artery diseases and atherosclerosis, one of the major diabetes related complications [30]. As shown in the Table 1, the levels of TC, TG, and LDL-c were elevated, in contrast to the HDL-c level which declined in alloxan- and steroid-induced diabetic rats compared to non-diabetic control rats. Increased levels of TC, TG and LDL-c, and decreased level of HDL-c highly increase the risk of coronary artery and cardiovascular diseases [34, 47]. Animals treated with PE and ME extracts of Ganoderma lucidum revert back the cholesterol and triglyceride levels about to normal levels. The mechanism behind this effect may be due to the fact that extract treatment promoted insulin secretion, and this increased insulin, thereby improving glucose utilization by the cells and decreasing free fatty acids immobilization from the fat depositions. Another possible mechanism may be that hypercholesterolemia is the result of increased intestinal absorption, as well as enhancement of cholesterol biosynthesis [33, 48].

From our thorough discussion, it is clear that Ganoderma lucidum contains bioactive compounds due to which its extract exhibited potential antihyperglycemic, insulin-sensitivity, and antihyperlipidemic activities in animal studies. Molecular profiling by HPLC, mass spectrophotometric analysis, nuclear magnetic resonance, etc. will be required. After successful identification and isolation of active ingredient(s) in this mushroom, the clinical trials for the efficacy and safety of the bioactive compound(s) will be required for the development of nutraceuticals or functional foods.

\section{CONCLUSION}

The results of our present investigation clearly demonstrate that petroleum ether and methanol extracts of Ganoderma lucidum (Gano mushroom) have the potential to reduce hyperglycemia with the enhanced glucose uptake, induce insulin production and secretion by pancreatic betacells, improves insulin sensitivity, and ameliorate lipid profile in alloxan and steroid-induced 
diabetic Long-Evans rats. Dietary intake or administration of extracts of Reishi mushroom will effective for the management of diabetes and hyperlipidemia.

Abbreviations Used: GL, Ganoderma lucidum; PEE, Petroleum ether extract; ME, Methanol extract; GL-PEE, Petroleum ether extract of Ganoderma lucidum; GL-PE, Methanol extract of Ganoderma lucidum; OGTT, Oral glucose tolerance test; LDL-c, low-density lipoprotein cholesterol; TC, Total cholesterol; TG, Triglycerides; HDL-c, High-density lipoprotein cholesterol; i.p., Intraperitoneal; HbA1c, Glycated hemoglobin; PPG, Post-prandial plasma glucose.

Competing Interests: The authors declare that they have no financial or intellectual conflicts of interest.

Authors' Contributions: All authors contributed to this study.

Acknowledgements and Funding: The authors would like to express their gratefulness to Primeasia University authority, Dhaka, Bangladesh, for financial support to conduct this research project. The authors are also thankful to the authority of Mushroom Development Institute, Ministry of Agriculture, Sobhanbag, Savar, Dhaka, Bangladesh, for kind donation and identification of Reishi mushroom (Ganoderma lucidum).

\section{REFERENCES}

1. American Diabetic Association (ADA). Diagnosis and classification of diabetes mellitus. Diab Care 2013; 36 (Suppl 1):S67-74.

2. Li F, Zhang Y, Zhong Z. Antihyperglycemic effect of ganoderma lucidum polysaccharides on streptozotocin-induced diabetic mice. Int J Mol Sci 2011; 12:61356145.

3. Suckling R, Gallagher H. Chronic kidney disease, diabetes mellitus and cardiovascular disease: risks and commonalities. JORC 2012; 38 (Suppl 1):4-11.

4. Habib SL, Rojna M. Diabetes and risk of cancer. ISRN Oncol 2013; 2013:583786.

5. American Diabetic Association (ADA). Cardiovascular disease and risk management. Diab Care 2015; 38 (Suppl 1):S49-57. doi: 10.2337/dc15-S011. doi: 10.2337/dc15-S011.

6. Jansson SP, Fall K, Brus O, Magnuson A, Wandell P, Ostgren CJ, et al. Prevalence and incidence of diabetes mellitus: a nationwide population-based pharmaco-epidemiological study in Sweden. Diab Med 2015; Feb 7, Ahead of Print. doi: 10.1111/dme.12716.

7. WHO. Facts and figures about diabetes, 10 facts about diabetes (2013). Available from http://www.who.int/features/factfiles/diabetes/en/ (Accessed on 7 June, 2015).

8. Monami M, Genovese S, Mannucci E. Cardiovascular safety of sulfonylureas: a metaanalysis of randomized clinical trials. Diab Obes Metab 2013; 15:938-953.

9. Hung AM, Roumie CL, Greevy RA, Liu X, Grijalva CG, Murff HJ, et al. Comparative effectiveness of incident oral antidiabetic drugs on kidney function. Kidney Int 2012; 81:698-706. 
10. Thakkar B, Aronis KN, Vamvini MT, Shields K, Mantzoros CS. Metformin and sulfonylureas in relation to cancer risk in type II diabetes patients: a meta-analysis using primary data of published studies. Metab Clin Exp 2013; 62:922-934.

11. Batra P, Sharma AK, Khajuria R. Probing Lingzhi or Reishi medicinal mushroom Ganoderma lucidum (higher Basidiomycetes): a bitter mushroom with amazing health benefits. Int J Med Mushrooms 2013; 15:127-143.

12. Pan D, Zhang D, Wu J, Chen C, Xu Z, Yang H, et al. Antidiabetic, antihyperlipidemic and antioxidant activities of a novel proteoglycan from ganoderma lucidum fruiting bodies on $\mathrm{db} / \mathrm{db}$ mice and the possible mechanism. PloS one 2013; 8:e68332.

13. Xu Z, Chen X, Zhong Z, Chen L, Wang Y. Ganoderma lucidum polysaccharides: immunomodulation and potential anti-tumor activities. Am J Chin Med 2011; 39:15-27.

14. Choi S, Nguyen VT, Tae N, Lee S, Ryoo S, Min BS, et al. Anti-inflammatory and heme oxygenase-1 inducing activities of lanostane triterpenes isolated from mushroom Ganoderma lucidum in RAW264.7 cells. Toxicol Appl Pharm 2014; 280:434-442.

15. Oluba OM, Adebisi KE, Eidangbe GO, Odutuga AA, Onyeneke EC. Modulatory effect of crude aqueous extract of Lingzhi or Reishi Medicinal Mushroom, Ganoderma lucidum (Higher Basidiomycetes), on hematological and antioxidant indices in Plasmodium berghei-infected mice. Int J Med Mushrooms 2014; 16:499-506.

16. Deepalakshmi K, Mirunalini S, Krishnaveni M, Arulmozhi V. In vitro and in vivo antioxidant potentials of an ethanolic extract of Ganoderma lucidum in rat mammary carcinogenesis. Chin J Nat Med 2013; 11:621-627.

17. Socala K, Nieoczym D, Grzywnowicz K, Stefaniuk D, Wlaz P. Evaluation of Anticonvulsant, Antidepressant-, and Anxiolytic-like Effects of an Aqueous Extract from Cultured Mycelia of the Lingzhi or Reishi Medicinal Mushroom Ganoderma lucidum (Higher Basidiomycetes) in Mice. Int J Med Mushrooms 2015; 17:209-218.

18. Matsuzaki H, Shimizu Y, Iwata N, Kamiuchi S, Suzuki F, lizuka H, et al. Antidepressant-like effects of a water-soluble extract from the culture medium of Ganoderma lucidum mycelia in rats. BMC Complement Altern Med 2013; 13:370.

19. Oluba OM, Olusola AO, Fagbohunka BS, Onyeneke E. Antimalarial and hepatoprotective effects of crude ethanolic extract of Lingzhi or Reishi medicinal mushroom, Ganoderma lucidum (W.Curt.:Fr.)P.Karst. (higher Basidiomycetes), in Plasmodium berghei-infected mice. Int J Med Mushrooms 2012; 14:459-466.

20. Vazirian M, Faramarzi MA, Ebrahimi SE, Esfahani HR, Samadi N, Hosseini SA, et al. Antimicrobial effect of the Lingzhi or Reishi medicinal mushroom, Ganoderma lucidum (higher Basidiomycetes) and its main compounds. Int J Med Mushrooms 2014; 16:77-84.

21. Wang J, Yuan Y, Yue T. Immunostimulatory activities of beta-d-glucan from Ganoderma Lucidum. Carbohydr Polym 2014; 102:47-54.

22. Shi Y, Cai D, Wang X, Liu X. Immunomodulatory effect of ganoderma lucidum polysaccharides (GLP) on long-term heavy-load exercising mice. Int J Vitam Nutr Res 2012; 82:383-390. 
23. Yue GG, Chan BC, Han XQ, Cheng L, Wong EC, Leung PC, et al. Immunomodulatory activities of Ganoderma sinense polysaccharides in human immune cells. Nutr Cancer 2013; 65:765-774.

24. Habijanic J, Berovic M, Boh B, Plankl M, Wraber B. Submerged cultivation of Ganoderma lucidum and the effects of its polysaccharides on the production of human cytokines TNF-alpha, IL-12, IFN-gamma, IL-2, IL-4, IL-10 and IL-17. New Biotechnol 2015; 32:85-95.

25. Li A, Shuai X, Jia Z, Li H, Liang X, Su D, et al. Ganoderma lucidum polysaccharide extract inhibits hepatocellular carcinoma growth by downregulating regulatory $\mathrm{T}$ cells accumulation and function by inducing microRNA-125b. J Transl Med 2015; 13:100.

26. Weng CJ, Yen GC. The in vitro and in vivo experimental evidences disclose the chemopreventive effects of Ganoderma lucidum on cancer invasion and metastasis. Clin Exp metastas 2010; 27:361-369.

27. Xiao C, Wu QP, Cai W, Tan JB, Yang XB, Zhang JM. Hypoglycemic effects of Ganoderma lucidum polysaccharides in type 2 diabetic mice. Arch Pharm Res 2012; 35:1793-1801.

28. Teng BS, Wang CD, Zhang D, Wu JS, Pan D, Pan LF, et al. Hypoglycemic effect and mechanism of a proteoglycan from ganoderma lucidum on streptozotocin-induced type 2 diabetic rats. Eur Rev Med Pharmacol Sci 2012; 16:166-175.

29. Zheng J, Yang B, Yu Y, Chen Q, Huang T, Li D. Ganoderma lucidum polysaccharides exert anti-hyperglycemic effect on streptozotocin-induced diabetic rats through affecting beta-cells. Comb Chem High T Scr 2012; 15:542-550.

30. Ulbricht C, Abrams TR, Bent S, Boon H, Costa D, Dacey C, et al. Reishi Mushroom (Ganoderma lucidum): Systematic Review by the Natural Standard Research Collaboration. J Soc Integ Oncol 2010; 8:148-159.

31. The State Pharmacopoeia Commission of P. R. China. State Pharmacopoeia Commission of the People's Republic of China. Beijing, China: Chemical Industry Press; 2000.

32. Wachtel-Galor S, Tomlinson B, Benzie FF. Ganoderma lucidum ('Lingzhi'), a Chinese medicinal mushroom: biomarker responses in a controlled human supplementation study. Br J Nutr 2004; 91:263-269.

33. Ahmed D, Kumar V, Verma A, Gupta PS, Kumar H, Dhingra V, et al. Antidiabetic, renal/hepatic/pancreas/cardiac protective and antioxidant potential of methanol/dichloromethane extract of Albizzia Lebbeck Benth. stem bark (ALEx) on streptozotocin induced diabetic rats. BMC Complement Altern Med 2014; 14:243.

34. Kumar V, Ahmed D, Gupta PS, Anwar F, Mujeeb M. Anti-diabetic, anti-oxidant and anti-hyperlipidemic activities of Melastoma malabathricum Linn. leaves in streptozotocin induced diabetic rats. BMC Complement Altern Med 2013; 13:222.

35. Balamurugan K, Nishanthini A, Mohan VR. Antidiabetic and antihyperlipidaemic activity of ethanol extract of Melastoma malabathricum Linn. leaf in alloxan induced diabetic rats. Asian Pac J Trop Biomed 2014; 4:S442-448. 
36. Kumarappan C, Mandal SC. Polyphenol extract of ichnocarpus frutescens leaves modifies hyperglycemia in dexamethasone (dex) treated rats. Indian J Physiol Pharmacol 2014; 58:441-445.

37. Sikarwar MS, Patil MB. Antidiabetic activity of Pongamia pinnata leaf extracts in alloxan-induced diabetic rats. Int J Ayurveda Res 2010; 1: 199-204.

38. Lino CDS, Diogenes JPL, Pereira BA, Faria RAPG, Neto MA, Alves RS, et al. Antidiabetic Activity of Bauhinia forficata Extracts in Alloxan-Diabetic Rats. Biol Pharm Bull 2004; 27: 125-127.

39. Toma A, Makonnen E, Mekonnen Y, Debella A, Adisakwattana S. Antidiabetic activities of aqueous ethanol and n-butanol fraction of Moringa stenopetala leaves in streptozotocin-induced diabetic rats. BMC Complement Altern Med 2015; 15:242.

40. Lenzen S. The mechanisms of alloxan- and streptozotocin-induced diabetes. Diabetologia 2008; 51:216-226.

41. Lansang MC, Hustak LK. Glucocorticoid-induced diabetes and adrenal suppression: how to detect and manage them. Cleve Clin J Med 2011; 78:748-756.

42. Ferris HA, Kahn CR. New mechanisms of glucocorticoid-induced insulin resistance: make no bones about it. J Clin Invest 2012; 122:3854-3857.

43. Schort RJ. Targeting Plasma Glucose: Preprandial Versus Postprandial. Clin Diabetes 2004; 22(4):169-172

44. Mohankumar SK, O'Shea T, McFarlane JR. Insulinotrophic and insulin-like effects of a high molecular weight aqueous extract of Pterocarpus marsupium Roxb. hardwood. $J$ Ethnopharmacol 2012; 141:72-79.

45. Nelson RH. Hyperlipidemia as a risk factor for cardiovascular disease. Prim care 2013; 40:195-211.

46. Miller M, Stone NJ, Ballantyne C, Bittner V, Criqui MH, Ginsberg HN, et al. Triglycerides and cardiovascular disease: a scientific statement from the American Heart Association. Circulation 2011; 123:2292-2333.

47. Nofer JR. Hyperlipidemia and cardiovascular disease: triglycerides - a revival of cardiovascular risk factor? Curr Opin Lipidol 2011; 22:319-321.

48. Subash Babu P, Prabuseenivasan S, Ignacimuthu S. Cinnamaldehyde--a potential antidiabetic agent. Phytomedicine 2007; 14:15-22. 